3. A. Hubert, R. Schäfer, Magnetic Domains (Springer, Berlin, 1998).

4. J. Raabe et al., J. Appl. Phys. 88, 4437 (2000).

5. T. Shinjo, T. Okuno, R. Hassdorf, K. Shigeto, T. Ono, Science 289, 930 (2000)

6. M. Pratzer et al., Phys. Rev. Lett. 87, 127201 (2001).

7. S. Heinze et al., Science 288, 1805 (2000)

8. A. Kubetzka, M. Bode, O. Pietzsch, R. Wiesendanger, Phys. Rev. Lett. 88, 057201 (2002).

9. The $\mathrm{Cr}$ film thickness dependent reorientation transition may be caused by the surface anisotropy that dominates at small thickness.

10. M. Bode, M. Getzlaff, R. Wiesendanger, Phys. Rev. Lett. 81, 4256 (1998).

11. O. Pietzsch, A. Kubetzka, M. Bode, R. Wiesendanger, Phys. Rev. Lett. 84, 5212 (2000)

12. D. Wortmann, S. Heinze, Ph. Kurz, G. Bihlmayer, S. Blügel, Phys. Rev. Lett. 86, 4132 (2001).
13. The spin polarizations of the tip and the sample are defined as $P_{\mathrm{S}, \mathrm{T}}(E) \equiv\left(n_{\mathrm{ST}}^{\downarrow}(E)-n_{\mathrm{S}, \mathrm{T}}(E)\right) /\left(n \downarrow-n_{\mathrm{S},}(E)+\right.$ $\left.n_{\mathrm{S}, \mathrm{T}}^{\uparrow}(E)\right)$ with $n_{\mathrm{S}, T}^{\downarrow}(E)$ and $n_{\mathrm{S}, \mathrm{T}}^{\uparrow}(E)$ being the density of 'states of majority and minority electrons.

14. O. Pietzsch, A. Kubetzka, D. Haude, M. Bode, R. Wiesendanger, Rev. Sci. Instr. 71, 424 (2000).

15. U. Gradmann, G. Liu, H. J. Elmers, M. Przybylski, Hyperfine Interactions 57, 1845 (1990).

16. The tiny bright spots randomly distributed in both images are caused by adsorbates that appear as dips in the topograph (Fig. 2A) and locally change the value of $C$ in Eq. 1; i.e., the signals are not of magnetic origin.

17. The simulations were performed with the OOMMF program, release 1.2 alpha 2 (http://math.nist.gov/ oommf/) using a lateral grid with a cell size of $1 \mathrm{~nm}^{2}$. The shape as well as the averaged island height of $8 \mathrm{~nm}$ was taken into account. Because the height of the Fe islands of about $8 \mathrm{~nm}$ is only 2.5 to 3 times larger than $\sqrt{A / K_{d}}$, the magnetization was assumed to be independent of the $z$ coordinate $(3,18)$.

18. U. Gradmann, J. Korecki, G. Waller, Appl. Phys. A 39, 101 (1986)

19. The small deviation between experimental and simulated data at $d_{v c}=10$ to $15 \mathrm{~nm}$ in the presence of external fields is caused by the lateral movement of the vortex core (Fig. 4B), which has not been considered in the simulations. At the rim of the $\mathrm{Fe}$ island, the magnetization tilts stronger into the direction of $H_{\text {ext }}$ than in the island center.

20. Financial support from the BMBF (grant no. 13N7647) and SFB 508 is gratefully acknowledged.

20 June 2002; accepted 4 September 2002

\title{
Microfluidic Large-Scale Integration
}

\author{
Todd Thorsen, ${ }^{1}$ Sebastian J. Maerkl, ${ }^{1}$ Stephen R. Quake ${ }^{2 *}$
}

\begin{abstract}
We developed high-density microfluidic chips that contain plumbing networks with thousands of micromechanical valves and hundreds of individually addressable chambers. These fluidic devices are analogous to electronic integrated circuits fabricated using large-scale integration. A key component of these networks is the fluidic multiplexor, which is a combinatorial array of binary valve patterns that exponentially increases the processing power of a network by allowing complex fluid manipulations with a minimal number of inputs. We used these integrated microfluidic networks to construct the microfluidic analog of a comparator array and a microfluidic memory storage device whose behavior resembles random-access memory.
\end{abstract}

In the first part of the 20th century, engineers faced a problem commonly called the "tyranny of numbers": there is a practical limit to the complexity of macroscopically assembled systems (1). Using discrete components such as vacuum tubes, complex circuits quickly became very expensive to build and operate. The ENIAC I, created at the University of Pennsylvania in 1946, consisted of 19,000 vacuum tubes, weighed 30 tons, and used $200 \mathrm{~kW}$ of power. The transistor was invented at Bell Laboratories in 1947 and went on to replace the bulky vacuum tubes in circuits, but connectivity remained a problem. Although engineers could in principle design increasingly complex circuits consisting of hundreds of thousands of transistors, each component within the circuit had to be hand-soldered - an expensive, laborintensive process. Adding more components to the circuit decreased its reliability, as even a single cold solder joint rendered the circuit useless.

In the late 1950s, Kilby and Noyce solved the "tyranny of numbers" problem for electronics by inventing the integrated circuit. By fab-

${ }^{1}$ Biochemistry and Molecular Biophysics Option, ${ }^{2} \mathrm{De}-$ partment of Applied Physics, California Institute of Technology, Pasadena, CA 91125, USA.

*To whom correspondence should be addressed. Email: quake@caltech.edu ricating all of the components out of a single semiconductor-initially germanium, then silicon-Kilby and Noyce created circuits consisting of transistors, capacitors, resistors, and their corresponding interconnects in situ, eliminating the need for manual assembly. By the mid1970s, improved technology led to the development of large-scale integration (LSI): complex integrated circuits containing hundreds to thousands of individual components.

Microfluidics offers the possibility of solving similar system integration issues for biology and chemistry. However, devices to date have lacked a method for a high degree of integration, other than simple repetition. Microfluidic systems have been shown to have potential in a diverse array of biological applications, including biomolecular separations (2-4), enzymatic assays $(5,6)$, the polymerase chain reaction $(6$, $7)$, and immunohybridization reactions $(8,9)$. These are excellent individual examples of scaled-down processes of laboratory techniques, but they are also stand-alone functionalities, comparable to a single component within an integrated circuit. The current industrial approach to addressing true biological integration has come in the form of enormous robotic fluidic workstations that take up entire laboratories and require considerable expense, space, and labor, reminiscent of the macroscopic approach to circuits consisting of massive vacuum tube-based arrays in the early 20 th century.

There are two basic requirements for a microfluidic LSI technology: monolithic microvalves that are leakproof and scalable, and a method of multiplexed addressing and control of these valves. We previously presented a candidate plumbing technology that allows fabrication of chips with monolithic valves made from the silicone elastomer polydimethylsiloxane (PDMS) (10). Here, we describe a microfluidic multiplexing technology and show how it can be used to fabricate silicone devices with thousands of valves and hundreds of individually addressable reaction chambers. As possible applications of fluidic LSI technology, we describe a chip that contains a high-density array of 1000 individually addressable picoliter-scale chambers that serves as a microfluidic memory storage device, and a second chip analogous to an array of 256 comparators.

Our microfluidic multiplexors are combinatorial arrays of binary valve patterns that increase the processing power of a network by allowing complex fluid manipulations with a minimal number of controlled inputs. Although simple microfluidic arrays can be designed in which each fluid channel is controlled by its own individual valve control channel, this nonintegrated strategy cannot be efficiently scaled up and thus faces problems similar to those encountered in pre-LSI electronic circuits. In contrast, multiplexors work as a binary tree (Fig. 1) and allow control of $n$ fluid channels with only $2 \log _{2} n$ control channels. We fabricated the devices with established multilayer soft lithography techniques (11), using two distinct layers. The "control" layer, which harbors all channels required to actuate the valves, is situated on top of the "flow" layer, which contains the network of channels being controlled. All biological assays and fluid manipulations are performed on the flow layer. A valve is created where a control channel crosses a flow channel. The resulting thin membrane in the junction between the two channels can be deflected by hydraulic actuation. Simultaneous addressing of multiple noncontiguous flow channels is accomplished by fabricating control 


\section{REPOR T S}

channels of varying width while keeping the dimension of the flow channel fixed $(100 \mu \mathrm{m}$ wide and $9 \mu \mathrm{m}$ high). The pneumatic pressure in the control channels required to close the flow channels scales with the width of the control channel, making it simple to actuate 100 $\mu \mathrm{m} \times 100 \mu \mathrm{m}$ valves at relatively low pressures $(\sim 40 \mathrm{kPa})$ without closing off the 50 $\mu \mathrm{m} \times 100 \mu \mathrm{m}$ crossover regions, which have a higher actuation threshold.

By using multiplexed valve systems, the power of the binary system becomes evident: Only 20 control channels are required to specifically address 1024 flow channels. This allows a large number of elastomeric microvalves to perform complex fluidic manipulations within these devices, and the interface between the device and the external environment is simple and robust. Introduction of fluid into these devices is accomplished through steel pins inserted into holes punched through the silicone. Unlike micromachined devices made out of hard materials with a high Young's modulus (12), silicone is soft and forms a tight seal around the input pins, readily accepting pressures of up to $300 \mathrm{kPa}$ without leakage. Computer-controlled external solenoid valves allow actuation of multiplexors, which in turn allow complex addressing of a large number of microvalves.

Using two multiplexors as fluidic design elements, we designed a microfluidic memory storage device with 1000 independent compartments and 3574 microvalves, organized as an addressable $25 \times 40$ chamber microarray (Fig. 2A). The large multiplexor valve systems allow each chamber of the matrix to be individually addressed and isolated, and only 22 outside control interconnects are needed. Fluid can be

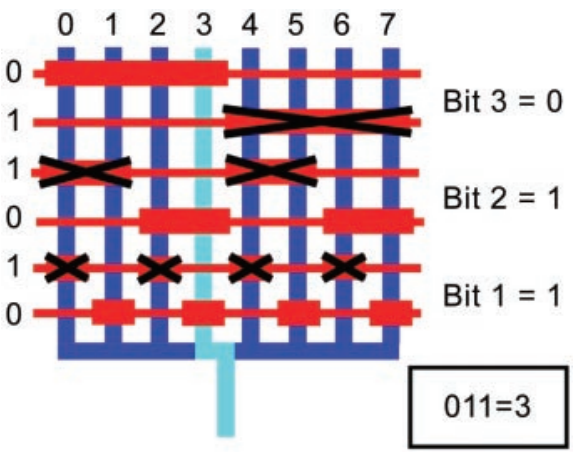

Fig. 1. Microfluidic multiplexor operational diagram. The blue lines represent flow channels containing the fluid of interest; the red lines represent control lines that can be hydraulically actuated. Valves are formed at the intersection of the wide part of a control channel with a flow channel. The actuation pressure is chosen so that only the wide membranes are fully deflected. Each combination of open and closed valves in the multiplexor selects for a single channel, so that $n$ flow channels can be addressed with only $2 \log _{2} n$ control channels. The pattern illustrated here has all channels closed except the cyan channel. loaded into the device through a single input port, after which control layer valves then act as gates to compartmentalize the array into chambers with a volume of $250 \mathrm{pl}\left(2.5 \times 10^{-4} \mu \mathrm{l}\right)$. Individual chamber addressing is accomplished through flow channels that run parallel to the sample chambers and use pressurized liquid under the control of the row and column multiplexors to flush the chamber contents to the output (Fig. 2B).

This device adds a level of complexity to previous microfluidic plumbing, in that there are two successive levels of control-the multiplexors actuate valve control lines, which in turn actuate the valves themselves. The design and mechanics of the microfluidic array are similar to random-access memory (RAM). Each set of multiplexors is analogous to a memory address register, mapping to a specific row or column in the matrix. Like dynamic RAM, the row and column multiplexors have unique functions. The row multiplexor is used for fluid
A Row

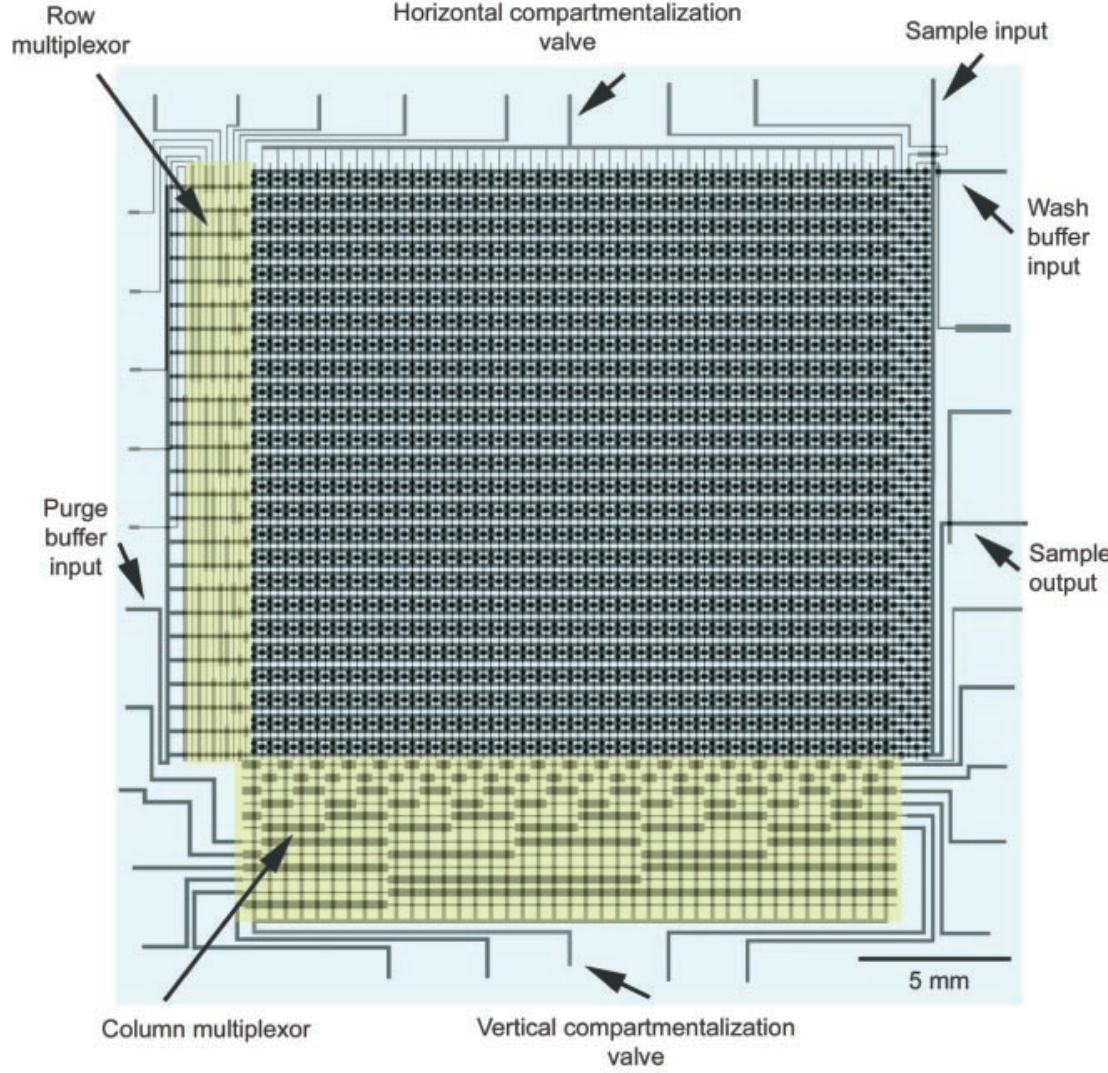

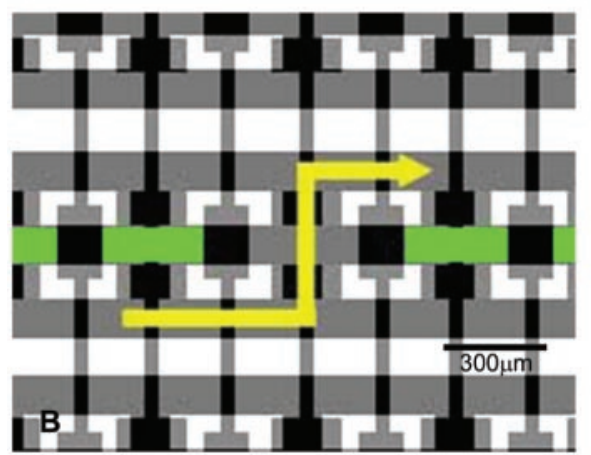

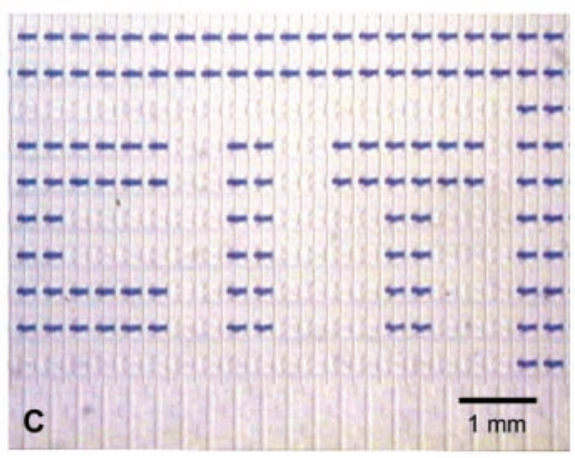

Fig. 2. (A) Mask design for the microfluidic memory storage device. The chip contains an array of $25 \times 40$ chambers, each of which has a volume of $\sim 250$ pl. Each chamber can be individually addressed using the column and row multiplexors. The contents of each memory location can be selectively programmed to be either blue dye (sample input) or water (wash buffer input). (B) Purging mechanics for a single chamber within a selected row of the chip. Each row contains three parallel microchannels. A specific chamber is purged as follows: (i) Pressurized fluid is introduced in the purge buffer input. (ii) The row multiplexor directs the fluid to the lower channel of the selected row. (iii) The column multiplexor releases the vertical valves of the chamber, allowing the pressurized fluid to flow through the chamber and purge its contents. (C) Demonstration of microfluidic memory display: Individual chambers are selectively purged to spell out "C I T". 


\section{REPOR T S}

trafficking; it directs the fluid responsible for purging individual compartments within a row and refreshes the central compartments (memory elements) within a row, analogous to a RAM word line. The column multiplexor acts in a fundamentally different manner, controlling the vertical input-output valves for specific central compartments in each row. The column multiplexor, located on the flow layer, begins to operate when the vertical containment valve on the control layer is pressurized to close off the entire array. It is activated with its valves deflected upward into the control layer to trap the pressurized liquid in the entire vertical containment valve array. A single column is then selected by the multiplexor, and the pressure on the vertical containment valve is released to open the specified column, allowing it to be rapidly purged by pressurized liquid in a selected row.

To demonstrate the functionality of the microfluidic memory storage device, we loaded

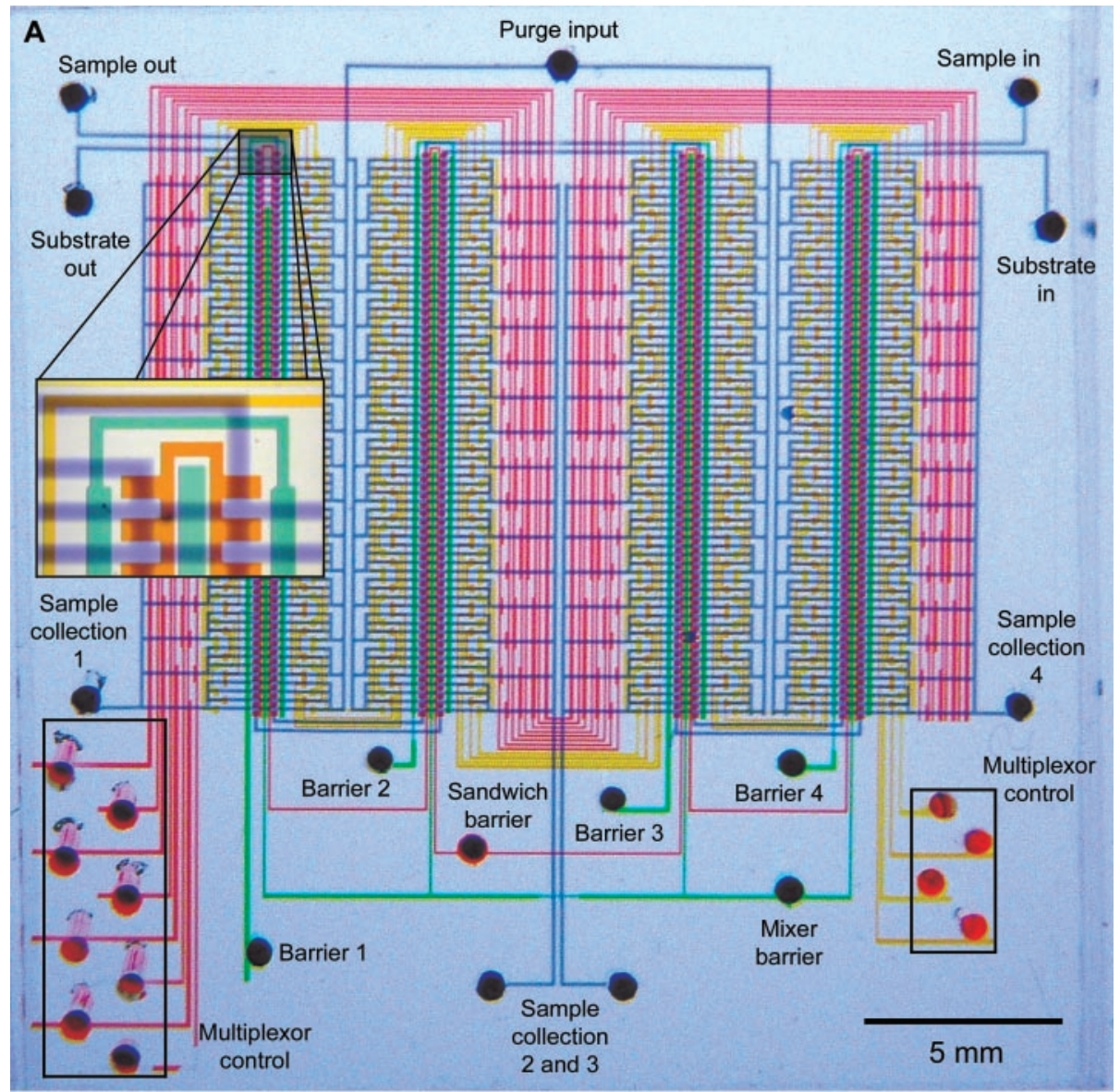

Fig. 3. (A) Optical micrograph of the microfluidic comparator chip. The various inputs have been loaded with food dyes to visualize the channels and subelements of the fluidic logic. (B) Set of optical micrographs showing a portion of the comparator in action. A subset of the chambers in a single column is imaged. Elastomeric microvalves enable each of the 256 chambers on the chip to be independently compartmentalized, mixed pairwise, and selectively purged with the blue and yellow solutions. the central memory storage chambers of each row with dye $(2.4 \mathrm{mM}$ bromophenol blue in sodium citrate buffer, $\mathrm{pH}$ 7.2) and proceeded to purge individual chambers with water to spell out "C I T". Because the readout is optical, this memory device also essentially functions as a fluidic display monitor (Fig. 2C). A key advantage of the plumbing display is that once the picture is set, the device consumes very little power.

We designed a second device containing 2056 microvalves (Fig. 3A), which is capable of performing more complex fluidic manipulations. In this case, two different reagents can be separately loaded, mixed pairwise, and selectively recovered, making it possible to perform distinct assays in 256 subnanoliter reaction chambers and then recover a particularly interesting reagent. The microchannel layout consists of four central columns in the flow layer consisting of 64 chambers per column, with each chamber containing $\sim 750 \mathrm{pl}$ of liquid after compartmentalization and mixing. Liquid is loaded into these columns through two separate inputs under low external pressure $(\sim 20$ $\mathrm{kPa}$ ), filling up the array in a serpentine fashion. Barrier valves on the control layer function to isolate the sample fluids from each other and from channel networks on the flow layer used to recover the contents of each individual chamber. These networks function under the control of a multiplexor and several other control valves (13). The elastomeric valves are analogous to electronic switches, serving as highimpedance barriers for fluidic trafficking. To demonstrate the device plumbing, we filled the fluid input lines with two dyes to illustrate the process of loading, compartmentalization, mixing, and purging of the contents of a single chamber within a column (Fig. 3B). Each of the 256 chambers on the chip can be individually addressed and its respective contents recovered for future analysis using only 18 connections to the outside world, illustrating the integrated nature of the microfluidic circuit.

We used this chip as a microfluidic compar-

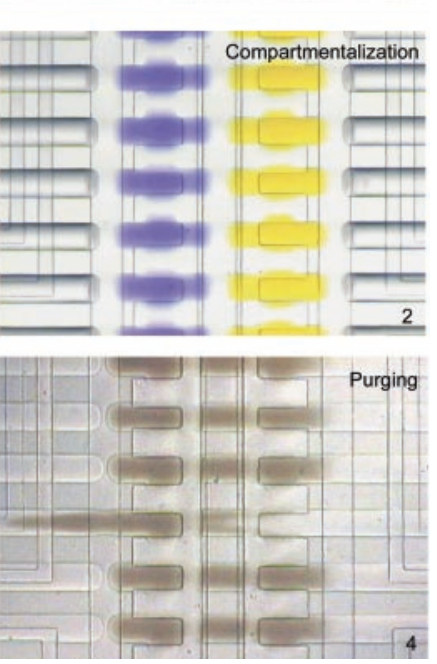

ator to test for the expression of a particular enzyme. A population of bacteria is loaded into the device, and a fluorogenic substrate system provides an amplified output signal in the form of a fluorescent product. An electronic comparator circuit is designed to provide a large output signal when the input signal exceeds a reference threshold value. An operational amplifier amplifies the input signal relative to the reference, forcing it to be high or low. In our microfluidic comparator, the nonfluorescent resorufin derivative Amplex Red functions as the reference signal. The input signal consists of a suspension of Escherichia coli expressing recombinant cytochrome c peroxidase (CCP); the enzyme serves as a chemical amplifier in the circuit (Fig. 4A). The cells and substrate are loaded into separate input channels with the central mixing barrier closed in each column and com- 


\section{R E P O R T S}

partmentalized exactly like the procedure illustrated for the blue and orange dyes. The cell dilution (1:1000 of confluent culture) creates a median distribution of $\sim 0.2$ cells per compartment, as verified by fluorescence microscopy. The barrier between the substrate and cell subcompartments is opened for a few minutes to allow substrate to diffuse into the compartments containing the cell mixture. The barrier is then closed to reduce the reaction volume and improve the signal/noise ratio for the reaction. After a 1-hour incubation at room temperature, the chip is scanned (excitation wavelength $\lambda_{\mathrm{ex}}=532 \mathrm{~nm}$, emission filter centered at $\lambda_{\mathrm{em}}=$ $590 \mathrm{~nm}$ with a $40-\mathrm{nm}$ bandwidth) with a modified DNA microarray scanner (GenePix 4000B, Axon Instruments, Union City, CA). The presence of one or more CCP-expressing cells in an individual chamber produces a strong amplified output signal, because Amplex Red is converted to the fluorescent compound resorufin while the signal in the compartments with no cells remains low (Fig. 4B). To verify that the output signal is a function of CCP activity, we performed a similar experiment using a heterogeneous mixture of $E$. coli expressing either CCP or enhanced green fluorescent protein (eGFP). The amplified output signal was only dependent on the number of CCP-expressing cells in an individual chamber (Fig. 4C).

Recovery from the chip can be accomplished by selecting a single chamber and then purging the contents to a collection output. Each column in the chip has a separate output, enabling a chamber from each column to be collected without cross-contamination. To illustrate the efficacy of the collection process, we loaded a dilute phosphate-buffered saline (PBS) solution of $E$. coli expressing eGFP into the chip. After compartmentalization, approximately every second chamber contained a bacterium. Using an inverted light microscope (Olympus IX50) equipped with a mercury lamp and GFP filter set, single eGFP cells were identified with a $20 \times$ objective and their respective chambers were purged. The purged cells were collected from the outputs with polyetheretherketone (PEEK) tubing, which has low cell adhesion properties. Isolations of single eGFP-expressing bacteria were confirmed by visualization of the collected liquid samples under a $40 \times$ oil immersion lens (using the fluorescence filter set) and by observations of single colony growth on LuriaBertani broth (LB) plates inoculated with the recovered bacteria. Because single molecules of DNA can be effectively manipulated in elastomeric microfluidic devices (14), it is possible that in future applications individual molecules or molecular clusters will be selected or manipulated in this fashion.

The performance of an electronic comparator is not ideal-for example, there is a finite noise floor, there are absolute voltage and cur- rent limitations, there are leakage currents at the inputs, and so forth. Some of these limits result from intrinsic properties of the materials used for the devices, whereas others depend on fabrication tolerances or design limitations. The performance of integrated fluidic circuits suffers from similar imperfections. Fluidic circuits fabricated from PDMS will not be compatible with all organic solvents - in particular, many of the nonpolar solvents present a problem. This issue can be addressed by the use of chemically resistant elastomers. Cross-contamination in microfluidic circuits is analogous to leakage currents in an electronic circuit and is a complex phenomenon. A certain amount of contamination will occur as a result of diffusion of small molecules through the elastomer itself. This effect is not an impediment with the organic dyes and other small molecules used in the examples in this work, but at some level and performance requirement it may become limiting. There are also surface effects due to nonspecific adhesion of molecules to the channel walls; these can be minimized by either passive $(15,16)$ or chemical $(17,18)$ modifications to the PDMS surface. Cross-contamination is also a design issue whose effects can be mitigated by the design of any particular circuit. In the 256-well comparator chip, we introduced a compensation scheme by which each of the
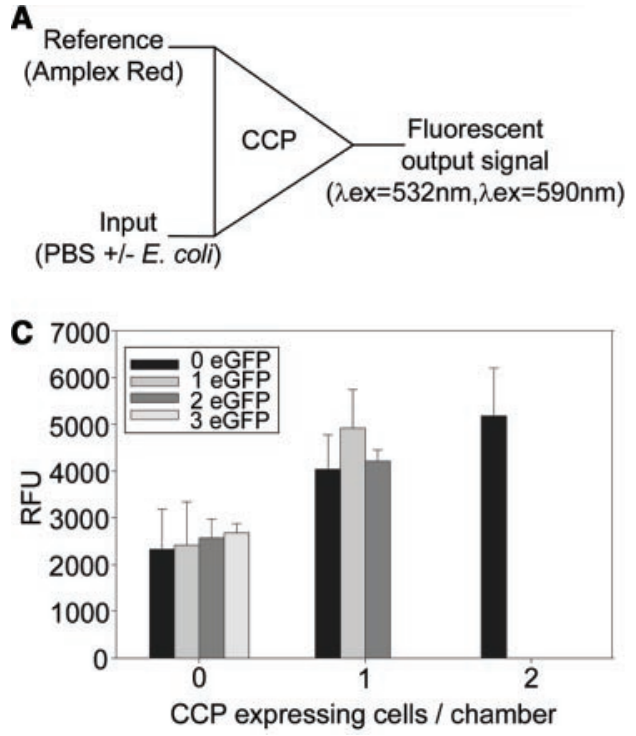

Fig. 4. (A) Schematic diagram of the microfluidic comparator logic using an enzyme and fluorogenic substrate. When an input signal chamber contains cells expressing the enzyme CCP, nonfluorescent Amplex Red is converted to the fluorescent product, resorufin. In the absence of CCP, the output signal remains low. (B) Scanned fluorescence image of the chip in comparator mode. Left side: Dilute solution of CCP-expressing $E$. coli in sterile PBS $(137 \mathrm{mM} \mathrm{NaCl}, 2.68 \mathrm{mM} \mathrm{KCl}, 10.1 \mathrm{mM}$ $\mathrm{Na}_{2} \mathrm{HPO}_{4}$, and $1.76 \mathrm{mM} \mathrm{KH}_{2} \mathrm{PO}_{4}, \mathrm{pH}$ 7.4) after mixing reaction with Amplex Red. Arrows indicate chambers containing single cells. Chambers without cells show low fluorescence. The converted product (resorufin) is clearly visible as green signal. Right side: Uncatalyzed Amplex Red substrate. (C) A micro-high-throughput screening comparator: Effect of heterogeneous mixture of eGFPexpressing control cells and CCP-expressing cells on output signal. The resorufin fluorescence measurement $\left(\lambda_{\text {ex }}=532 \mathrm{~nm}, \lambda_{\mathrm{em}}=590 \mathrm{~nm}\right)$ was made in individual comparator chambers containing $E$. coli cells expressing either eGFP or CCP. There is a strong increase in signal only when CCP-expressing cells are present, with little effect on the signal from eGFP-expressing cells. The vertical axis is relative fluorescence units (RFU); error bars represent one standard deviation from the median RFU. 
grated circuits can be usefully transferred to chemical and biochemical analysis and processing in microfluidic devices.

The two devices presented here illustrate that complex fluidic circuits with nearly arbitrary complexity can be fabricated using microfluidic LSI. The rapid, simple fabrication procedure combined with the powerful valve multiplexing can be used to design chips for many applications, ranging from high-throughput screening applications to the design of new liquid display technology. The scalability of the process makes it possible to design robust microfluidic devices with even higher densities of functional valve elements, so that the ultimate complexity and application are limited only by one's imagination.

\section{References and Notes}

1. T. R. Reid, The Chip: How Two Americans Invented the Microchip and Launched a Revolution (Simon \& Schuster, New York, 1984)

2. A. G. Hadd, S. C. Jacobson, J. M. Ramsey, Anal. Chem. 71, 5206 (1999)

3. D. J. Harrison et al., Science 261, 895 (1993).

4. P. C. H. Li, D. J. Harrison, Anal. Chem. 69, 1564 (1997).

5. A. G. Hadd, D. E. Raymond, J. W. Haliwell, S. C. Jacobson, S. C. Ramsey, Anal. Chem. 69, 3407 (1997).

6. E. T. Lagally, I. Medintz, R. A. Mathies, Anal. Chem. 73, 565 (2001).

7. J. Khandurina et al., Anal. Chem. 72, 2995 (2000).

8. E. Eteshola, D. Leckband, Sens. Actua. B 72, 129 (2001).

9. J. Wang, A. Ibanez, M. P. Chatrathi, A. Escarpa, Anal. Chem. 73, 5323 (2001).

10. M. A. Unger, H.-P. Chou, T. Thorsen, A. Scherer, S. R. Quake, Science 288, 113 (2000).

11. Master molds for the microfluidic channels are made by spin-coating positive photoresist (Shipley SJR 5740) on silicon (9 $\mu \mathrm{m}$ high) and patterning them with high-resolution (3386 dpi) transparency masks. The channels on the photoresist molds are rounded at $120^{\circ} \mathrm{C}$ for $20 \mathrm{~min}$ to create a geometry that allows full valve closure. The devices were fabricated by bonding together two layers of two-part cure silicone (Dow Corning Sylgard 184) cast from the photoresist molds. The bottom layer of the device, containing the flow channels, is spin-coated with 20:1 part $A: B$ Sylgard at $2500 \mathrm{rpm}$ for $1 \mathrm{~min}$. The resulting silicone layer is $\sim 30 \mu \mathrm{m}$ thick. The top layer of the device, containing the control channels, is cast as a thick layer ( $\sim .5 \mathrm{~cm}$ thick) with 5:1 part A:B Sylgard using a separate mold. The two layers are initially cured for $30 \mathrm{~min}$ at $80^{\circ} \mathrm{C}$. Control channel interconnect holes are then punched through the thick layer (released from the mold), after which it is sealed, channel side down, on the thin layer, aligning the respective channel networks. Bonding between the assembled layers is accomplished by curing the devices for an additional 45 to $60 \mathrm{~min}$ at $80^{\circ} \mathrm{C}$. The resulting multilayer devices are cut to size and mounted on RCA cleaned No. 1, $25 \mathrm{~mm}$ square glass coverslips, or onto coverslips spin-coated with 5:1 part A:B Sylgard at 5000 $\mathrm{rpm}$, and cured at $80^{\circ} \mathrm{C}$ for $30 \mathrm{~min}$, followed by incubation at $80^{\circ} \mathrm{C}$ overnight.

12. L. Buchaillot, E. Farnault, M. Hoummady, H. Fujita, Jpn. J. Appl. Phys. 2 36, L794 (1997).

13. The control channels are dead end-filled with water before actuation with pneumatic pressure; the compressed air at the ends of the channels is forced into the bulk porous silicone. This procedure eliminates gas transfer into the flow layer upon valve actuation, as well as evaporation of the liquid contained in the flow layer.

14. H. P. Chou, C. S. Spence, A. Scherer, S. R. Quake, Proc. Natl. Acad. Sci. U.S.A. 96, 11 (1999)

15. V. Linder, E. Verpoorte, W. Thormann, N. F. de Rooij, H. Sigrist, Anal. Chem. 73, 4181 (2001).
16. T. Yang, S. Jung, H. Mao, P. S. Cremer, Anal. Chem. 73, 165 (2001).

17. D. C. Duffy, J. C. MacDonald, O. J. A. Schueller, G. M. Whitesides, Anal. Chem. 70, 4974 (1998).

18. B. A. Grzybowski, R. Haag, N. Bowden, G. M. Whitesides, Anal. Chem. 70, 4645 (1998).

19. We thank M. Enzelberger, C. Hansen, M. Adams, and
M. Unger for helpful discussions. Supported in part by Army Research Office grant DAAD19-00-1-0392 and by the DARPA Bioflips program.

5 August 2002; accepted 17 September 2002 Published online 26 September 2002; 10.1126/science.1076996

Include this information when citing this paper.

\title{
Self-Assembly of Highly Phosphorylated Silaffins and Their Function in Biosilica Morphogenesis
}

\author{
Nils Kröger, ${ }^{1}$ Sonja Lorenz, ${ }^{2}$ Eike Brunner, ${ }^{2}$ Manfred Sumper ${ }^{1 *}$
}

\begin{abstract}
Silaffins are uniquely modified peptides that have been implicated in the biogenesis of diatom biosilica. A method that avoids the harsh anhydrous hydrogen fluoride treatment commonly used to dissolve biosilica allows the extraction of silaffins in their native state. The native silaffins carry further posttranslational modifications in addition to their polyamine moieties. Each serine residue was phosphorylated, and this high level of phosphorylation is essential for biological activity. The zwitterionic structure of native silaffins enables the formation of supramolecular assemblies. Time-resolved analysis of silica morphogenesis in vitro detected a plastic silaffin-silica phase, which may represent a building material for diatom biosilica.
\end{abstract}

Diatoms are unicellular, eukaryotic algae that produce a wide variety of nanopatterned silica structures in a genetically controlled manner (1). Biosilica morphogenesis is an extremely rapid process that is accomplished under mild physiological conditions, thus exceeding the capabilities of present-day materials engineering. Elucidating the molecular mechanisms of biosilica formation is therefore expected to help researchers devise new synthetic routes to nanostructured silica materials $(2,3)$.

Recently, silaffins and long-chain polyamines have been identified as constituents of biosilica and shown to accelerate silica formation from a monosilicic acid solution in vitro $(4,5)$. On the basis of the physicochemical properties of polyamines, a phase separation model has been proposed that is able to explain the nanopatterning of biosilica (6). Silaffins are peptides that carry numerous posttranslational modifications. The silaffins from Cylindrotheca fusiformis contain lysine residues that are linked by their $\varepsilon$-amino groups to long-chain polyamines (7). It is due to this modification that silaffin peptides can precipitate silica nanospheres at mildly acidic $\mathrm{pH}$ conditions (4), which likely represent the physiologically relevant $\mathrm{pH}$ range $(8)$.

Previously, the extraction and purification of silaffins has been achieved by the treatment of diatom shells with anhydrous hydrogen fluoride

'Lehrstuhl Biochemie I, '2nstitut für Biophysik und Physikalische Biochemie, Universität Regensburg, 93053 Regensburg, Germany.

*To whom correspondence should be addressed. Email: manfred.sumper@vkl.uni-regensburg.de
(HF), a treatment known to cleave O-glycosidic and phosphate ester bonds (9). However, silaffins contain a high percentage of hydroxyamino acids, candidates for posttranslational modifications. Therefore, the silaffins extracted to date may have lost functional modifications of hydroxyamino acids. To address this concern and to be able to study silica precipitation activities of native silaffins, we developed a gentler method for silaffin extraction. We found that native silaffins carry additional modifications that proved to be essential for biosilica formation.

It has previously been shown that an acidic aqueous solution of ammonium fluoride is capable of dissolving diatom biosilica (10) by converting silica into soluble ammonium hexafluorosilicate. When C. fusiformis cell walls are treated with an aqueous solution of ammonium fluoride at $\mathrm{pH}=5$, the complete set of silaffins and long-chain polyamines is solubilized. The apparent molecular masses of all silaffin species present in the aqueous ammonium fluoride extract are shifted toward higher molecular masses, indicating the existence of HF-labile modifications (11). An abundant peptide (apparent molecular mass of $6.5 \mathrm{kD}$ ) was purified from the ammonium fluoride extract (Fig. 1, lane 1) (12). After an additional treatment with anhydrous HF, this peptide exhibited a much higher electrophoretic mobility and comigrated in SDS-polyacrylamide gel electrophoresis (SDS-PAGE) with silaffin-1A (Fig. 1, lanes 2,3$)$, which has previously been characterized from HF-extracted cell walls (4). $\mathrm{NH}_{2}-$ terminal amino acid sequencing of this material resulted in the sequence SSXXSGSYSGS (G, Gly; S, Ser; Y, Tyr; X, modified lysine residue) 